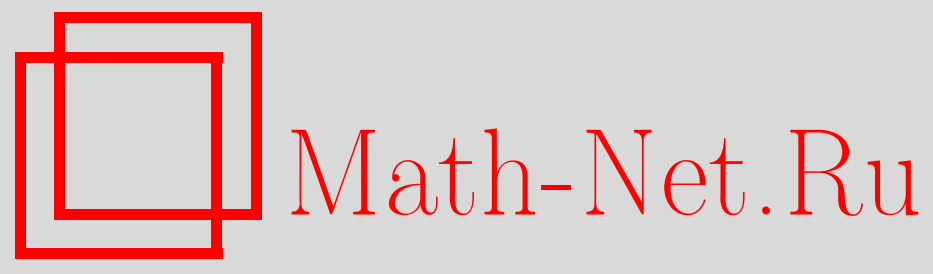

Б. Е. Бродский, Б. С. Дарховский, Минимаксные последовательные тесты проверки многих сложных гипотез. I, Теория вероятн. и ее примен., 2007, том 52, выпуск 4, 625-643

DOI: https://doi.org/10.4213/tvp1526

Использование Общероссийского математического портала MathNet.Ru подразумевает, что вы прочитали и согласны с пользовательским соглашением

http://www . mathnet.ru/rus/agreement

Параметры загрузки:

IP : 54.81 .137 .203

26 апреля 2023 г., $11: 32: 30$

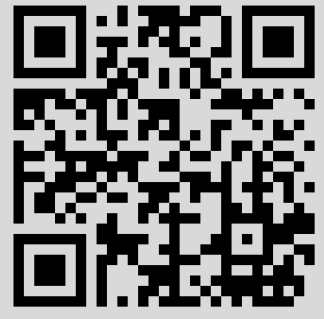




\section{МИНИМАКСНЫЕ ПОСЛЕДОВАТЕЛЬНЫЕ ТЕСТЫ ПРОВЕРКИ МНОГИХ СЛОЖНЫХ ГИПОТЕЗ. I}

В статье рассматривается задача последовательной проверки нескольких сложных гипотез. Каждая из гипотез описывается плотностью, зависящей от параметра, который может принадлежать одному из непересекаюшихся множеств. Предлагаются новые критерии качества для последовательных односторонних и многосторонних тестов и устанавливаются неасимптотические априорные нижние границы для предложенных критериев. Найдены последовательные тесты, использующие процедуру взятия минимакса по параметрическим множествам для последовательного отношения правдоподобия, на которых асимптотически достигаются априорные нижние границы критериев. Все доказательства отнесены в часть II.

Ключевые слова и фразы: проверка сложных гипотез, последовательные тесты.

1. Введение. Прошло более 60 лет с тех пор, как Вальд выдвинул свои основополагающие идеи в проблеме последовательной проверки двух простых гипотез (см., например, [1], [2]). Былю приложено много усилий для обобщения последовательного теста отношения правдоподобия Вальда (ПТОП) на различные случаи проверки сложных гипотез.

Приведем описание классической процедуры Вальда в удобном для дальнейших ссылок виде. Пусть $x_{1}, x_{2}, \ldots$ - последовательность независимых и одинаково распределенных случайных величин с общим распределением Р. Пусть $c_{0}>0, c_{1}>0, c \stackrel{\text { def }}{=}\left(c_{0}, c_{1}\right)$ - фиксированные константы. Введем функцию $g(x)=2 \mathbb{I}\left(x>c_{1}\right)-\mathbb{I}\left(x \geqslant-c_{0}\right)$ (здесь и далее символ $\mathbb{I}(A)$ означает индикатор множества $A$ ) и положим $\psi\left(x_{1}, \ldots, x_{n}\right) \stackrel{\text { def }}{=} \sum_{k=1}^{n} \ln \left(f_{1}\left(x_{k}\right) / f_{0}\left(x_{k}\right)\right)$ (здесь и далее $f_{i}$ - плотность распределения (п.р.) $\mathbf{P}_{i}$ по отношению к некоторой общей доминирующей мере $\nu, i=0,1)$. Введем решаюшую функиию $\mathscr{D}(n) \stackrel{\text { def }}{=} g\left(\psi\left(x_{1}, \ldots, x_{n}\right)\right)$

* Центральный экономико-математический институт РАН, Нахимовский пр-т, 47, 117218 Москва, Россия; e-mail: bbrodsky@yandex.ru

** Институт системного анализа РАН, пр-т 60-летия Октября, 9, 117312 Москва, B-312, Россия; e-mail: darbor@isa.ru 
и момент остановки (м.о.) $T(c)=\inf \{n: \mathscr{D}(n) \neq-1\}$ (здесь и далее мы полагаем $\inf (\varnothing)=+\infty)$.

Тогда м.о. в двусторонней прочедуре Вальда для проверки нулевой гипотезы $\mathbf{H}_{0}: \mathbf{P}=\mathbf{P}_{0}$ против альтернативы $\mathbf{H}_{1}: \mathbf{P}=\mathbf{P}_{1}$ есть м.о. $T(c)$, а заключительное решение имеет вид: если $\mathscr{D}(T(c))=1$, то принимается гипотеза $\mathbf{H}_{1}$, если $\mathscr{D}(T(c))=0$, то принимается гипотеза $\mathbf{H}_{0}$.

Заметим, что можно ввести в рассмотрение односторонние тесты Вальда. А именно, положим $g_{0}(x)=-\mathbb{I}\left(x \geqslant-c_{0}\right), g_{1}(x)=2 \mathbb{I}\left(x>c_{1}\right)-1$ и введем решающие функции и м.о. для односторонних тестов Вальда: $\mathscr{D}_{0}(n) \stackrel{\text { def }}{=} g_{0}\left(\psi\left(x_{1}, \ldots, x_{n}\right)\right), \mathscr{D}_{1}(n) \stackrel{\text { def }}{=} g_{1}\left(\psi\left(x_{1}, \ldots, x_{n}\right)\right), \quad T_{0}\left(c_{0}\right)=$ $\inf \left\{n: \mathscr{D}_{0}(n)=0\right\}, T_{1}\left(c_{0}\right)=\inf \left\{n: \mathscr{D}_{1}(n)=1\right\}$. Тогда заключительные решения для односторонних тестов Вальда будут такими: если $\mathscr{D}_{0}\left(T_{0}\left(c_{0}\right)\right)=0$, то принимается гипотеза $\mathbf{H}_{0}$, если $\mathscr{D}_{1}\left(T_{1}\left(c_{1}\right)\right)=1$, то принимается гипотеза $\mathbf{H}_{1}$. Очевидно, что $T(c)=\min \left(T_{0}\left(c_{0}\right), T_{1}\left(c_{1}\right)\right)$ и $\mathscr{D}(n)=\max \left(\mathscr{D}_{0}(n), \mathscr{D}_{1}(n)\right)$.

Как было показано Вальдом и Вольфовицем [3], ПТОП Вальда оптимален в следующем смысле: среди всех последовательных тестов, для которых м.о. имеет конечное математическое ожидание при обеих гипотезах и вероятности ошибок удовлетворяют соотношениям

$$
\mathbf{P}_{0}\left\{\text { отвергнуть } \mathbf{H}_{0}\right\} \leqslant \alpha, \quad \mathbf{P}_{1}\left\{\text { отвергнуть } \mathbf{H}_{1}\right\} \leqslant \beta,
$$

тест ПТОП (в том случае, когда можно подобрать константы $c_{0}$ и $c_{1}$ так, чтобы для вероятностей ошибок в (1.1) выполнялись точные равенства) минимизирует средний объем выборки как при одной, так и при другой гипотезе.

В 1959-1960 гг. были многочисленные попытки применить тест ПТОП к различным задачам проверки сложных гипотез. Одна из наиболее известных постановок была предложена Кифером и Вейссом [4]: проверить сложную гипотезу $\mathbf{H}_{0}: \theta<\theta_{0}$ против альтернативы $\mathbf{H}_{1}: \theta>\theta_{1}$, где $\theta$ - параметр п.р. наблюдений. Оказалось, что средний объем выборки в зоне безразличия $\theta_{0}<\theta<\theta_{1}$ может быть значительно больше, чем соответствующие объемы выборки для точек $\theta_{0}$ и $\theta_{1}$. Кифером и Вейссом была поставлена проблема: найти последовательный тест, который минимизирует средний объем выборки $\mathrm{E}_{\theta^{*}} T$ для любой заданной параметрической точки $\theta=\theta^{*}$ п.р. $f_{\theta}(x)$ при ограничениях на вероятности ошибок для фиксированных точек $\theta=\theta_{0}$ и $\theta=\theta_{1}$. Асимптотические решения задачи Кифера-Вейсса были найдены в работах [5], [6] и [7].

Асимптотический анализ последовательных тестов для сложных гипотез изучался на основе априорных теоретических границ для среднего объема выборки, которые были получены Вальдом и Хёфдингом [8]. В [9] было показано, что асимптотическим решением задачи Кифера-Вейсса 
является двусторонний ПТОП вида

$$
\widehat{N}=\inf \left\{n: \prod_{i=1}^{n} \frac{f_{\theta^{*}}\left(x_{i}\right)}{f_{\theta_{0}}\left(x_{i}\right)} \geqslant A_{0} \text { или } \prod_{i=1}^{n} \frac{f_{\theta^{*}}\left(x_{i}\right)}{f_{\theta_{1}}\left(x_{i}\right)} \geqslant A_{1}\right\},
$$

если $\max \left(A_{0}, A_{1}\right) \rightarrow \infty$. В этой же работе с помощью компьютерного моделирования было установлено, что $\mathbf{E}_{\theta^{*}} \widehat{N}$ близко к нижней границе Хёфдинга.

Во всех описанных постановках проблемы проверки сложных гипотез точки $\theta_{0}$ и $\theta_{1}$ для вероятностей ошибок были фиксированы.

В работах [10] и [11] рассматривалась задача последовательной проверки сложных гипотез, параметризованных двумерным вектором $(\theta, \eta)$, для независимых и одинаково распределенных случайных величин с плотностью из экспоненциального семейства. Здесь $\eta-$ мешающий параметр, а гипотезы имеют следующий вид: $\mathbf{H}_{0}: \theta=\theta_{0}, \eta \in Q$, и $\mathbf{H}_{1}: \theta=\theta_{1}, \eta \in Q$, где $Q$ - некоторое множество. Было предложено использовать отношение правдоподобия, в котором неизвестный параметр $\eta$ заменялся его оценкой максимального правдоподобия (ОМП) [10] или двумя ОМП, соответствующими параметрам $\theta_{0}$ и $\theta_{1}$ [11] (очевидно, что для простых гипотез в отсутствие мешаюшего параметра получается классический тест Вальда). Асимптотические свойства этих статистик и их численные характеристики изучались в [12]. В [13] (см. также [14]) было показано, что оптимальные свойства статистик [10] и [11] (аналогичные тесту Вальда) не сохраняются без предположения контигуальности для параметров $\theta_{0}$ и $\theta_{1}$. Сравнительный анализ асимптотических свойств теста Вальда и теста Неймана-Пирсона проведен в работе [15].

В работе [16] была рассмотрена задача проверки многих сложных гипотез в присутствии зоны безразличия для неизвестного параметра $\theta$. Были предложены два варианта последовательных тестов, удовлетворяющих заданным ограничениям на вероятности ошибок. Эти тесты обобщали более ранние результаты [17] и [18]. Было показано, что при достаточно общих условиях предложенные тесты обладают асимптотической оптимальностью первого порядка (для среднего объема выборки) для всех значений $\theta$, включая зону безразличия, при условии, что вероятности ошибок стремятся к нулю. Результаты работы [16] (как в дискретном, так и в непрерывном времени) получены в предположении, что п.р. принадлежит экспоненциальному семейству.

В работах [19], [20] исследовалась асимптотическая оптимальность ПТОП для многих простых гипотез и независимых наблюдений. Рассматривались два типа тестов. Первый тип тестов был предложен ранее в [21]-[23]. Второй тип тестов был предложен в [24], [17]. Случай зависимых наблюдений рассматривался в [25] и [26] для задачи последовательного обнаружения целей. 
Недавно в [27] были предложены асимптотические нижние границы для среднего объема выборки в задаче последовательной проверки многих гипотез для зависимых наблюдений, которые совпадают с ранее найденными (см. [28]) границами для независимого случая, если вероятности ошибок стремятся к нулю. Полученные нижние границы затем были использованы для анализа обобшенного теста отношения правдоподобия в задачах последовательной проверки многих гипотез и задачах о «разладке».

В этой статье мы предлагаем новые критерии качества для односторонних и многосторонних последовательных тестов проверки многих сложных гипотез. Для предлагаемых критериев установлены нижние неасимптотические гранищы и указываются тесты, на которых эти границы достигаются (асимптотически) при увеличении порогов.

Идею наших критериев качества проше всего пояснить для односторонних последовательных тестов проверки двух простых гипотез. Пусть $d_{i}(n), i=0,1$, - произвольные решаюшие функции односторонних последовательных тестов, т.е. измеримые функции наблюдений, принимающие в момент времени $n$ значения $i$ (принять гипотезу $\mathbf{H}_{i}$ ) и -1 (продолжить наблюдения), и пусть

$$
\nu_{i}=\inf \left\{n: d_{i}(n) \neq-1\right\}, \quad i=0,1,
$$

- м.о. наблюдений.

Односторонние процедуры можно рассматривать как процедуры решения вырожденной задачи о «разладке». Например, односторонняя процедура, порождаемая правилом $d_{0}(n)$, соответствует следующей вырожденной задаче о разладке: основная гипотеза - $\mathbf{H}_{1}-$ отвечает «неразлаженной» последовательности, гипотеза $\mathbf{H}_{0}$ отвечает «разладке», произошедшей в самьй первый момент наблюдений.

Тогда односторонняя процедура направлена на скорейшее обнаружение исходной «разладки» т.е. если «разладки» нет (верна гипотеза $\mathbf{H}_{1}$ ), то наблюдения должны продолжаться как можно дольше, а если есть «разладка» (т.е. просто верна с самого начала гипотеза $\mathbf{H}_{\mathbf{0}}$ ), то наблюдения должны остановиться как можно быстрее.

Рассмотрим величины

$$
\Gamma_{0}\left(\nu_{0}\right) \stackrel{\text { def }}{=} \sup _{n} \mathbf{P}_{1}\left\{d_{0}(n)=0\right\}, \quad \Gamma_{1}\left(\nu_{1}\right) \stackrel{\text { def }}{=} \sup _{n} \mathbf{P}_{0}\left\{d_{1}(n)=1\right\},
$$

которые можно назвать максимальными вероятностями ошибочных решений для односторонних прочедур.

Оказывается, что для всех м.о. вида (1.2) (с конечными математическими ожиданиями по каждой мере) имеют место неасимптотические неравенства

$$
\mathscr{K}_{0}\left(\nu_{0}\right) \stackrel{\text { def }}{=} \frac{\mathbf{E}_{0} \nu_{0}}{\left|\ln \Gamma_{0}\left(\nu_{0}\right)\right|} \geqslant\left|1+O\left(\frac{1}{\ln \Gamma_{0}\left(\nu_{0}\right)}\right)\right|\left(\int \ln \frac{f_{0}(x)}{f_{1}(x)} f_{0}(x) d x\right)^{-1}
$$




$$
\begin{aligned}
& \stackrel{\text { def }}{=}\left|1+O\left(\frac{1}{\ln \Gamma_{0}\left(\nu_{0}\right)}\right)\right| \mathbf{I}_{0}^{-1} \\
\mathscr{K}_{1}\left(\nu_{1}\right) & \stackrel{\text { def }}{=} \frac{\mathbf{E}_{1} \nu_{1}}{\left|\ln \Gamma_{1}\left(\nu_{1}\right)\right|} \geqslant\left|1+O\left(\frac{1}{\ln \Gamma_{1}\left(\nu_{1}\right)}\right)\right|\left(\int \ln \frac{f_{1}(x)}{f_{0}(x)} f_{1}(x) d x\right)^{-1} \\
& \stackrel{\text { def }}{=}\left|1+O\left(\frac{1}{\ln \Gamma_{1}\left(\nu_{1}\right)}\right)\right| \mathbf{I}_{1}^{-1} .
\end{aligned}
$$

При этом для процедуры Вальда имеют место равенства

$$
\lim _{c_{0} \rightarrow \infty} \mathscr{K}_{0}\left(T_{0}\left(c_{0}\right)\right)=\mathbf{I}_{0}^{-1}, \quad \lim _{c_{1} \rightarrow \infty} \mathscr{K}_{1}\left(T_{1}\left(c_{1}\right)\right)=\mathbf{I}_{1}^{-1} .
$$

Теперь рассмотрим произвольную двустороннюю последовательную процедуру с решающей функцией $d(n)=0,1,-1$ (последнее значение соответствует решению о продолжении наблюдений). Тогда

$$
\begin{gathered}
\tau_{0} \stackrel{\text { def }}{=} \inf \{n: d(n) \neq-1, d(n)=0\}=\inf \{n: d(n)=0\}, \\
\tau_{1} \stackrel{\text { def }}{=} \inf \{n: d(n) \neq-1, d(n)=1\}=\inf \{n: d(n)=1\}
\end{gathered}
$$

и момент остановки наблюдений $\tau$ двусторонней процедуры имеет вид

$$
\tau=\inf \{n: d(n) \neq-1\}=\min \left(\tau_{0}, \tau_{1}\right)
$$

\section{Положим}

$$
\begin{array}{ll}
\gamma_{0}(\tau) \stackrel{\text { def }}{=} \sup _{n} \mathbf{P}_{1}\{d(n)=0\}, & \beta_{10} \stackrel{\text { def }}{=} \inf _{n} \mathbf{P}_{1}\{d(n)=0\}, \\
\gamma_{1}(\tau) \stackrel{\text { def }}{=} \sup _{n} \mathbf{P}_{0}\{d(n)=1\}, & \beta_{01} \stackrel{\text { def }}{=} \inf _{n} \mathbf{P}_{0}\{d(n)=1\} .
\end{array}
$$

Оказывается, что для любого м.о. указанного вида (с конечными математическими ожиданиями по каждой мере) справедливы неасимптотические неравенства

$$
\begin{aligned}
\mathscr{K}_{0}(\tau) \stackrel{\text { def }}{=} \frac{\mathbf{E}_{0} \tau}{\left|\ln \gamma_{0}(\tau)\right|} \geqslant \mathrm{I}_{0}^{-1}\left(1-\gamma_{1}+\left(1-\gamma_{1}\right) \frac{\ln \left(1-\gamma_{1}\right)}{\left|\ln \gamma_{0}\right|}+\frac{\beta_{01}}{\left|\ln \gamma_{0}\right|} \ln \frac{\beta_{01}}{1-\beta_{10}}\right. \\
\left.+O\left(\frac{1}{\left|\ln \gamma_{0}(\tau)\right|}\right)\right)
\end{aligned}
$$$$
\mathscr{K}_{1}(\tau) \stackrel{\text { def }}{=} \frac{\mathbf{E}_{1} \tau}{\left|\ln \gamma_{1}(\tau)\right|} \geqslant \mathbf{I}_{1}^{-1}\left(1-\gamma_{0}+\left(1-\gamma_{0}\right) \frac{\ln \left(1-\gamma_{0}\right)}{\left|\ln \gamma_{1}\right|}+\frac{\beta_{10}}{\left|\ln \gamma_{1}\right|} \ln \frac{\beta_{10}}{1-\beta_{01}}\right.
$$$$
\left.+O\left(\frac{1}{\left|\ln \gamma_{1}(\tau)\right|}\right)\right)
$$

Если $c \stackrel{\text { def }}{=}\left(c_{0}, c_{1}\right) \rightarrow \infty$, то для вальдовского м.о. одновременно вblполнень равенства

$$
\lim _{c \rightarrow \infty} \mathscr{K}_{0}(T(c))=\mathbf{I}_{0}^{-1}, \quad \lim _{c \rightarrow \infty} \mathscr{K}_{1}(T(c))=\mathbf{I}_{1}^{-1},
$$


т.е. если ввести векторньй критерий качества теста (или м.о.)

$$
\mathscr{K}(\tau) \stackrel{\text { def }}{=}\left\{\mathscr{K}_{0}(\tau), \mathscr{K}_{1}(\tau)\right\}
$$

то для вальдовской прочедуры этот векторный критерий асимптотически достигает своей априорной нижней границы при стремлении $\kappa$ бесконечности «большого параметра»с.

Поясним смысл введенных критериев. Очевидно, что для односторонних последовательных процедур с решающими функциями $d_{i}(n)$, $i=0,1$, имеем соответственно

$$
\left\{\nu_{0}=n\right\} \subset\left\{d_{0}(n)=0\right\}, \quad\left\{\nu_{1}=n\right\} \subset\left\{d_{1}(n)=1\right\} .
$$

Поэтому

$$
\sup _{n} \mathbf{P}_{1}\left\{\nu_{0}=n\right\} \leqslant \Gamma_{0}\left(\nu_{0}\right), \quad \sup _{n} \mathbf{P}_{0}\left\{\nu_{1}=n\right\} \leqslant \Gamma_{1}\left(\nu_{1}\right) .
$$

Известно [31], что если случайная величина $\xi$ с целочисленными положительными значениями такова, что $\sup _{n} \mathbf{P}\{\xi=n\} \leqslant \alpha$, то $\mathbf{E} \xi \geqslant$ $(2 \alpha)^{-1}(1+o(1))$ при $\alpha \rightarrow 0$ и эта оценка точна.

Поэтому м.о. для односторонних правил, удовлетворяющие условию $\left|\ln \Gamma_{0}\left(\nu_{0}\right)\right| \geqslant|\ln a|$ или $\left|\ln \Gamma_{1}\left(\nu_{1}\right)\right| \geqslant|\ln b|$, обладают (при достаточно малых $a$ и $b$ ) свойством $\ln \mathbf{E}_{1} \nu_{0} \geqslant 0.9|\ln a|$ или $\ln \mathbf{E}_{0} \nu_{1} \geqslant 0.9|\ln b|$.

Как уже отмечалось, односторонние процедуры есть процедуры решения вырожденной задачи о «разладке». Но в задачах о «разладке» часто используется следующая постановка: минимизировать среднее время запаздывания при условии, что среднее время до ложной тревоги больше заданной константы.

Имея это в виду, целесообразно для односторонних тестов ставить такие задачи:

$$
\begin{aligned}
& \mathbf{E}_{0} \nu_{0} \longrightarrow \text { inf по множеству }\left\{\nu_{0}: \ln \mathbf{E}_{1}\left(\nu_{0}\right) \geqslant 0.9 u\right\} \stackrel{\text { def }}{=} Q_{0}(u), \\
& \mathbf{E}_{1} \nu_{1} \longrightarrow \text { inf по множеству }\left\{\nu_{1}: \ln \mathbf{E}_{0}\left(\nu_{1}\right) \geqslant 0.9 v\right\} \stackrel{\text { def }}{=} Q_{1}(v) .
\end{aligned}
$$

\section{Положим}

$$
G_{0}(u) \stackrel{\text { def }}{=}\left\{\nu_{0}:\left|\ln \Gamma_{0}\left(\nu_{0}\right)\right| \geqslant u\right\}, \quad G_{1}(v) \stackrel{\text { def }}{=}\left\{\nu_{1}:\left|\ln \Gamma_{1}\left(\nu_{1}\right)\right| \geqslant v\right\} .
$$

В силу указанных выше оценок

$$
G_{0}(u) \subseteq Q_{0}(u), \quad G_{1}(v) \subseteq Q_{1}(v)
$$

Однако при $u \rightarrow \infty, v \rightarrow \infty$ множества $Q_{0}(u)$ и $Q_{1}(v)$ сужаются, а вместе с ними сужаются и множества $G_{0}(u)$ и $G_{1}(v)$. Поэтому асимптотически, для больших средних времен до ложной тревоги, рассмотрение задач

$$
\begin{aligned}
& \mathbf{E}_{0} \nu_{0} \longrightarrow \text { inf по множеству }\left\{\nu_{0}:\left|\ln \Gamma_{0}\left(\nu_{0}\right)\right| \geqslant u\right\}=G_{0}(u), \\
& \mathbf{E}_{1} \nu_{1} \longrightarrow \text { inf по множеству }\left\{\nu_{1}:\left|\ln \Gamma_{1}\left(\nu_{1}\right)\right| \geqslant v\right\}=G_{1}(v) .
\end{aligned}
$$

в содержательном смысле эквивалентно рассмотрению задач (1.3). 
Поэтому задачи (1.4) мы будем называть задачами в стандартной постановке.

Обратим внимание теперь на то обстоятельство, что все разумные правила остановки, известные из литературы, обычно зависят от некоторого «большого параметра» $c$, который обладает тем свойством, что при его стремлении к бесконечности вероятность ложного решения стремится к нулю. Очень часто таким параметром является порог (как в процедуре Вальда), но это не обязательно.

Покажем, что задачи в стандартной постановке (1.4) можно заменить следуюшими задачами:

$$
\begin{aligned}
& \mathscr{K}_{0}\left(\nu_{0}\right)=\frac{\mathbf{E}_{0} \nu_{0}}{\left|\ln \Gamma_{0}\left(\nu_{0}\right)\right|} \longrightarrow \text { inf }, \\
& \mathscr{K}_{1}\left(\nu_{1}\right)=\frac{\mathbf{E}_{1} \nu_{1}}{\left|\ln \Gamma_{1}\left(\nu_{1}\right)\right|} \longrightarrow \text { inf },
\end{aligned}
$$

т.е. если найдены решения в задачах минимизации вида (1.5), то эти решения - при правильном выборе большого параметра - будут близки (по значению) к оптимальном решениям в задачах вида (1.4).

Пусть $\nu_{0}^{*}(c)$ - решение (первой) задачи (1.5) при большом параметpe $c$. Предположим, что существует предел

$$
\lim _{c \rightarrow \infty} \frac{\left|\ln \Gamma_{0}\left(\nu_{0}^{*}(c)\right)\right|}{c}=\beta>0
$$

(на самом деле достаточно, чтобы этим свойством обладал нижний предел), и пусть при $c>r$

$$
c(\beta-\varepsilon) \leqslant\left|\ln \Gamma_{0}\left(\nu_{0}^{*}(c)\right)\right| \leqslant c(\beta+\varepsilon) .
$$

Будем считать, что число $u$ в (1.4) достаточно велико, а именно, $u>r(\beta-\varepsilon)$. Выберем $\tilde{c}=u /(\beta-\varepsilon)$. Тогда, во-первых, $\tilde{c}>r$, а вовторых,

$$
\widetilde{c}(\beta+\varepsilon) \geqslant\left|\ln \Gamma_{0}\left(\nu_{0}^{*}(\widetilde{c})\right)\right| \geqslant \widetilde{c}(\beta-\varepsilon)=u,
$$

т.е. $\nu_{0}^{*}(\widetilde{c}) \in G_{0}(u)$. Поэтому

$$
\inf _{\nu_{0} \in G_{0}(u)} \mathbf{E}_{0} \nu_{0} \leqslant \mathbf{E}_{0} \nu_{0}^{*}(\widetilde{c}) .
$$

С другой стороны, поскольку для $\nu_{0} \in G_{0}(u)$

$$
\frac{\mathbf{E}_{0} \nu_{0}}{\left|\ln \Gamma_{0}\left(\nu_{0}\right)\right|} \leqslant \frac{\mathbf{E}_{0} \nu_{0}}{u}
$$

Tо

$$
\begin{aligned}
\inf _{\nu_{0} \in G_{0}(u)} \mathbf{E}_{0} \nu_{0} & \geqslant u \inf _{\nu_{0} \in G_{0}(u)} \frac{\mathbf{E}_{0} \nu_{0}}{\left|\ln \Gamma_{0}\left(\nu_{0}\right)\right|} \geqslant u \inf _{\nu_{0}} \frac{\mathbf{E}_{0} \nu_{0}}{\left|\ln \Gamma_{0}\left(\nu_{0}\right)\right|} \\
& =u \frac{\mathbf{E}_{0} \nu_{0}^{*}(\widetilde{c})}{\mid \ln \Gamma_{0}\left(\nu_{0}^{*}(\widetilde{c})\right)} \geqslant u \frac{\mathbf{E}_{0} \nu_{0}^{*}(\widetilde{c})}{\widetilde{c}(\beta+\varepsilon)}=\frac{\beta-\varepsilon}{\beta+\varepsilon} \mathbf{E}_{0} \nu_{0}^{*}(\widetilde{c})
\end{aligned}
$$


т.е. из (1.6) и (1.7) следует, что м.о. $\nu_{0}^{*}(\widetilde{c})$ является $\varepsilon$-оптимальным в задаче (1.4).

Из приведенных рассмотрений следует, что критерии $\mathscr{K}_{0}\left(\nu_{0}\right)$ и $\mathscr{K}_{1}\left(\nu_{1}\right)$ при достаточно малых вероятностях принятия ошибочных решений представляют собой отношение (в подходяшем масштабе) среднего времени до верного решения $к$ наихудшему (т.е. наименьшему) среднему времени до ложного решения.

Для двусторонней последовательной процедуры с решающей функцией $d(n)$ при проверке двух простых гипотез положим

$$
\tau_{0} \stackrel{\text { def }}{=} \inf \{n: d(n)=0\}, \quad \tau_{1} \stackrel{\text { def }}{=} \inf \{n: d(n)=1\} .
$$

Тогда момент остановки наблюдений $\tau$ имеет вид

$$
\tau=\inf \{n: d(n) \neq-1\}=\min \left(\tau_{0}, \tau_{1}\right) .
$$

Поскольку $\left\{\tau_{0}=n\right\} \subset\{d(n)=0\}$ и $\left\{\tau_{1}=n\right\} \subset\{d(n)=1\}$, то

$$
\begin{aligned}
& \sup _{n} \mathbf{P}_{1}\left\{\tau_{0}=n\right\} \leqslant \sup _{n} \mathbf{P}_{1}\{d(n)=0\}=\gamma_{0}(\tau), \\
& \sup _{n} \mathbf{P}_{0}\left\{\tau_{1}=n\right\} \leqslant \sup _{n} \mathbf{P}_{0}\{d(n)=1\}=\gamma_{1}(\tau)
\end{aligned}
$$

и, следовательно, как и выше, при достаточно малых $\gamma_{0}(\tau), \gamma_{1}(\tau)$

$$
\ln \mathbf{E}_{1} \tau_{0} \geqslant 0.9\left|\ln \gamma_{0}(\tau)\right|, \quad \ln \mathbf{E}_{0} \tau_{1} \geqslant 0.9\left|\ln \gamma_{1}(\tau)\right|
$$

Поэтому критерии

$$
\mathscr{K}_{0}(\tau)=\frac{\mathbf{E}_{0} \tau}{\left|\ln \gamma_{0}(\tau)\right|} \sim \frac{\mathbf{E}_{0} \tau}{\ln \mathbf{E}_{1} \tau_{0}}, \quad \mathscr{K}_{1}(\tau)=\frac{\mathbf{E}_{1} \tau}{\left|\ln \gamma_{1}(\tau)\right|} \sim \frac{\mathbf{E}_{1} \tau}{\ln \mathbf{E}_{0} \tau_{1}},
$$

как и ранее, представляют собой отношение (в подходящем масштабе) среднего времени до верного решения $\kappa$ наихудшему среднему времени до ложного решения.

Но поскольку теперь решений об остановке наблюдений и принятии одной из гипотез два, то и характеризовать такие процедуры следует векторным критерием.

Итак, в ситуации двух простых гипотез целесообразно в качестве критериев оптимальности для односторонних тестов использовать критерий $\mathscr{K}_{0}\left(\nu_{0}\right)$ для проверки гипотезы $\mathbf{H}_{0}$ и критерий $\mathscr{K}_{1}\left(\nu_{1}\right)$ для проверки гипотезы $\mathbf{H}_{1}$, а для двусторонних тестов применять векторньий критерий

$$
\mathscr{K}(\tau) \stackrel{\text { def }}{=}\left\{\mathscr{K}_{0}(\tau), \mathscr{K}_{1}(\tau)\right\}
$$

причем, как было указано выше, односторонние и двусторонняя процедуры Вальда асимптотически оптимальны в смысле введенных критериев. 
Аналогичным образом рассматривается и ситуация проверки сложных гипотез. Для иллюстрации ограничимся случаем одностороннего последовательного теста проверки двух сложных гипотез. А именно, пусть $\mathscr{P}_{0}=\left\{\mathbf{P}_{\theta_{0}}\right\}_{\theta_{0} \in \Theta_{0}}, \mathscr{P}_{1}=\left\{\mathbf{P}_{\theta_{1}}\right\}_{\theta_{1} \in \Theta_{1}}, \Theta_{0} \cap \Theta_{1}=\varnothing$, - два параметрических семейства распределений, заданных своими п.р. $f\left(\theta_{0}, x\right), f\left(\theta_{1}, x\right)$ и соответствуюших гипотезам $\mathbf{H}_{0}$ и $\mathbf{H}_{1}$. Пусть $d_{1}\left(n, c_{1}\right)$ - решаюшая функция (зависящая от «большого параметра» $c_{1}$ ) одностороннего последовательного теста проверки гипотезы $\mathbf{H}_{1}$. Для произвольного $\theta_{1} \in \Theta_{1}$ можно указать «наименее благоприятную альтернативу» $\theta_{0}\left(\theta_{1}\right) \stackrel{\text { def }}{=} \theta_{0}(\cdot)$. Эта альтернатива соответствует такой точке $\theta_{0}(\cdot) \in \Theta_{0}$, для которой расстояние Кульбака-Лейблера между распределениями $\mathbf{P}_{\theta_{1}}$ и $\mathbf{P}_{\theta_{0}(\cdot)}$ будет минимальным (точные определения будут даны ниже).

Пусть

$$
\Gamma_{1}\left(c_{1}\right)=\sup _{n} \mathbf{P}_{\theta_{0}(\cdot)}\left\{d_{1}\left(n, c_{1}\right)=1\right\}, \quad \nu_{1}\left(c_{1}\right)=\inf \left\{n: d_{1}\left(n, c_{1}\right)=1\right\} \text {. }
$$

В этом случае предлагаемый критерий качества м.о. $\nu_{1}\left(c_{1}\right)$ можно записать так:

$$
\mathscr{K}\left(\nu_{1}\left(c_{1}\right), \theta_{1}\right) \stackrel{\text { def }}{=} \frac{\mathbf{E}_{\theta_{1}} \nu_{1}\left(c_{1}\right)}{\left|\ln \Gamma_{1}\left(c_{1}\right)\right|} .
$$

Оказывается, что этот критерий имеет неасимптотическую априорную нижнюю гранииу, которая, если максимальная вероятность ложного решения стремится к нулю, стремится к константе, зависящей лишь от пары $\left(\theta_{0}(\cdot), \theta_{1}\right)$, а не от конкретного теста. Отсюда следует, что предел этого критерия при $c_{1} \rightarrow \infty$ (если он существует) имеет априорную нижнюю границу, равную указанной константе.

Имея в виду указанный выше смысл «большого параметра», можно сказать, что среднее время до ложного решения $\mathbf{E}_{\theta_{0}(\cdot)} \nu_{1}\left(c_{1}\right)$ имеет порядок $\Gamma_{1}\left(c_{1}\right)^{-1}$ (при $\left.c_{1} \rightarrow \infty\right)$. Поэтому мы получаем, что предельная величина предлагаемого критерия есть отношение (в подходяшем масштабе) среднего времени до верного решения $\kappa$ наихудшему среднему времени до ложного решения для произвольной аипотезы из семейства $\mathscr{P}_{1}$ и ее наименее благоприятной альтернативь из семейства $\mathscr{P}_{0}$.

Кроме того, как и для простых гипотез, тест, который оптимален в смысле предлагаемого критерия, будет оптимальным и для стандартной постановки задачи при правильном выборе «большого параметра».

На наш взгляд, предлагаемый критерий (который, очевидно, следует минимизировать) в содержательном смысле не хуже, ием обычно используемые критерии, а именно, среднее время до верного решения при заданных вероятностях ошибок заключительного решения и, более того, наилучший в смысле этого критерия тест будет (как продемонстрировано выше), при правильном выборе «большого параметра», наилучшим и в стандартной постановке. Предлагаемые критерии качества для 
последовательной проверки гипотез представляют также и методологический интерес, поскольку соответствуюшие экстремальные проблемы ставятся как проблемы безусловной минимизации.

В силу наличия априорной нижней границы для предлагаемых критериев можно ставить задачи поиска асимптотически оптимальных (при $c \rightarrow \infty)$ односторонних и многосторонних тестов для последовательной проверки сложных гипотез, которые будут обладать тем свойством, что на них достигаются нижние границы в априорных неравенствах. Здесь мы имеем ситуацию, вполне аналогичную неравенству Рао-Крамера для оценок параметра. Оказывается, что асимптотически оптимальные тесты получаются при помощи операции минимакса по параметрическим множествам.

Для случая конечного числа простых гипотез наши асимптотически оптимальные тесты, по сушеству, совпадают с тестами, предложенными в [27]. Однако для случая многих сложных гипотез наши тесты отличаются от тестов из [27], поскольку не используют априорное вероятностное распределение на параметрическом множестве. Заметим также, что результат работы [27] для случая многих сложных гипотез базируется на нереализуемом условии (см. условие (25) в [27]).

Идеологически наши асимптотически оптимальные тесты согласуются с (технически более сложными) тестами, предложенными в [16], но нам не требуется предположение о принадлежности распределений экспоненциальному семейству, а наши априорные неравенства являются неасимптотическими.

Структура статьи такова. В п. 2 формулируются основные предположения, в п. 3 рассмотрены односторонние тесты, приведена априорная нижняя граница для новых критериев качества и указаны асимптотически оптимальные методы. В п. 4 рассматриваются многосторонние последовательные тесты, приводятся априорные нижние границы для новых критериев качества и указывается асимптотически оптимальный тест. Все доказательства приводятся в ч. II.

Результаты статьи (при соответствующей переформулировке) остаются справедливыми для случайных последовательностей с перемешиванием и могут быть обобшены для непараметрического описания гипотез. Мы здесь не останавливаемся на этом. Упрощенная версия результатов работы (случай двух сложных гипотез) анонсирована в [32].

2. Предположения. Пусть $I \stackrel{\text { def }}{=}\{0,1, \ldots, k-1\}, \theta=\left(\theta_{0}, \theta_{1}, \ldots\right.$, $\left.\theta_{k-1}\right), k \geqslant 2, \theta \in \Theta, \theta_{i} \in \Theta_{i}$, где $\Theta-$ параметрическое множество, принадлежашее некоторому открытому множеству $U$ в конечномерном пространстве, $\Theta=\bigcup_{i} \Theta_{i}, \Theta_{i} \cap \Theta_{j}=\varnothing, i \neq j$. Наблюдается последовательность независимых случайных векторов $\left\{\xi_{k}\right\}_{k=1}^{\infty}$ с п.р. по отношению к некоторой $\sigma$-конечной мере $\mu$, равной $f\left(\theta_{i}, x\right), \theta_{i} \in \Theta_{i}$, если 
справедлива гипотеза $\mathbf{H}_{i}$. Плотность распределения $f$ известна и определена для всех значений параметра из $U$. Всюду далее $\mathbf{P}_{\theta_{i}}$ и $\mathbf{E}_{\theta_{\boldsymbol{i}}}$ будут обозначать меру и математическое ожидание, соответствующие последовательности $\left\{\xi_{k}\right\}_{k=1}^{\infty}$ с фиксированным значением параметра $\theta_{i}$.

Всюду далее мы предполагаем, что выполняются следующие условия.

У с л о в и е 2.1. Множество $\Theta$ компакт.

У с л о в и е 2.2. $\mu\left\{x: f\left(a_{1}, x\right) \neq f\left(a_{2}, x\right)\right\}>0$, если $a_{1} \neq a_{2}$.

У с л о в и е 2.3. Для $\mu$-п.в. $x$ функции $f\left(\theta_{i}, x\right), i \in I$, непрерывны и положительны по $\theta_{i} \in \Theta_{i}$.

Всюду ниже для любого фиксированного $\theta_{i} \in \Theta_{i}, i \in I$, будем использовать обозначения $\mathscr{U}_{i} \stackrel{\text { def }}{=} \Theta \backslash \Theta_{i}, \mathscr{Z}_{i} \stackrel{\text { def }}{=} \Theta_{i} \times \mathscr{U}_{i}=\left\{z_{i}\right\}$.

У с л о в и е 2.4. Функции $\left(\mathbf{E}_{\theta_{i}} \ln \left(f\left(\theta_{i}, \cdot\right) / f(u, \cdot)\right)\right)^{-1}$ непрерывны по $u \in \mathscr{U}_{i}$ для любого $\theta_{i} \in \Theta_{i}, i \in I$.

У с л о в и е 2.5 (равномерное условие Крамера). При $|t|<H$ для любого $i \in I$

$$
\sup _{\theta_{i}^{*} \in \Theta_{i}} \sup _{z_{i} \in \mathscr{Z}_{i}} \int\left(\frac{f\left(\theta_{i}, x\right)}{f\left(u_{i}, x\right)}\right)^{t} f\left(\theta_{i}^{*}, x\right) \mu(d x)<\infty .
$$

У с л о в и е 2.6. Для любого $i \in I$

$$
0<\inf _{\theta_{i}^{*} \in \Theta_{i}} \inf _{z_{i} \in \mathscr{Z}_{i}} \mathbf{E}_{\theta_{i}^{*}} \ln \frac{f\left(\theta_{i}, \cdot\right)}{f(u, \cdot)}, \quad \sup _{\theta_{i}^{*} \in \Theta_{i}} \sup _{z_{i} \in \mathscr{Z}_{i}} \mathbf{E}_{\theta_{i}^{*}} \ln \frac{f(u, \cdot)}{f\left(\theta_{i}, \cdot\right)}<0 .
$$

У с л о в и е 2.7. Для любых $z_{i} \in \mathscr{Z}_{i}, u^{*} \in \mathscr{U}_{i}$ функция

$$
\varkappa_{i}\left(t, z_{i}, u^{*}\right) \stackrel{\text { def }}{=} \ln \int\left(\frac{f\left(\theta_{i}, x\right)}{f(u, x)}\right)^{t} f\left(u^{*}, x\right) \mu(d x)
$$

имеет только два нуля: 0 и $t_{i}^{*}\left(z_{i}, u^{*}\right)>0$, функция $t_{i}^{*}\left(\cdot, u^{*}\right)$ непрерывна для любого $u^{*} \in \mathscr{U}_{i}$ и $\min _{z_{i} \in \mathscr{Z}_{i}} t_{i}^{*}\left(z_{i}, u^{*}\right)>0$.

3 а м е ч а и е 2.1. Условия компактности и непрерывности могут быть ослаблены, но мы на этом не останавливаемся для упрощения изложения.

3. Односторонние тесты: основные результаты. Односторонняя последовательная процедура проверки гипотезы $\mathbf{H}_{i}, i \in I$, представляет собой выбор из двух возможных решений: прекратить наблюдения и принять гипотезу $\mathbf{H}_{i}$ или продолжить наблюдения.

Пусть $c_{i}$ - некоторый «большой параметр» (мы будем исследовать асимптотическую оптимальность последовательных тестов при $\left.c_{i} \rightarrow \infty\right)$. Пусть $d_{i}\left(n, c_{i}\right)$ - решающая функция односторонней последовательной процедуры для проверки гипотезы $\mathbf{H}_{i}$, т.е. измеримая функция наблюдений $\left(x_{1}, \ldots, x_{n}\right)$, принимающая в момент времени $n$ значение $i$, если наблюдения прекрашаются и принимается гипотеза $\mathbf{H}_{i}$, и значение -1 , если наблюдения продолжаются. 
Пусть

$$
\nu_{i}\left(c_{i}\right) \stackrel{\text { def }}{=} \inf \left\{n: d_{i}\left(n, c_{i}\right)=i\right\}, \quad i \in I,
$$

- м.о., соответствующие односторонним тестам.

Введем следующие классы м.о. (и соответствующих односторонних тестов):

$$
\mathscr{M} \stackrel{\text { def }}{=}\left\{\nu_{i}\left(c_{i}\right) \text { : для любого конечного } c \sup _{\theta_{j} \in \Theta_{j}} \mathrm{E}_{\theta_{j}} \nu_{i}\left(c_{i}\right)<\infty \forall i, j \in I\right\} .
$$

Рассмотрим односторонние тесты, порожденные м.о. из множества $\mathscr{M}$. Пусть $\theta_{i}$ - произвольная точка из множества $\Theta_{i}$. Отправляясь от точки $\theta_{i}$, определим точку $u_{i}\left(\theta_{i}\right) \stackrel{\text { def }}{=} u_{i}(\cdot)$ как аргумент максимума следующей функции:

$$
\max _{u \in \Theta \backslash \Theta_{i}}\left(\mathbf{E}_{\theta_{i}} \ln \frac{f\left(\theta_{i}, x\right)}{f(u, x)}\right)^{-1} \stackrel{\text { def }}{=} \mathrm{I}_{i}^{-1}\left(\theta_{i}\right)
$$

Если множество точек максимума этой функции состоит более чем из одной точки, то выбираем произвольную точку соответствующего множества в качестве $u_{i}(\cdot)$ (ниже будет видно, что выбор такой точки не влияет на оптимальное правило).

Если точка $\theta_{i}$ является истинным значением параметра п.р. при гипотезе $\mathbf{H}_{i}$, то точка $u_{i}(\cdot)$ соответствует наименее благоприятной альтернативе для этого случая: расстояние Кульбака-Лейблера $\operatorname{dist}\left(\mathbf{P}_{\theta_{i}}, \mathbf{P}_{u_{i}(\cdot)}\right)$ между распределениями $\mathbf{P}_{\theta_{i}}, \mathbf{P}_{u_{i}(\cdot)}$ минимально в силу определения $u_{i}(\cdot)$. Введем

$$
\Gamma_{i}\left(c_{i}\right)=\sup _{n} \mathbf{P}_{u_{i}(\cdot)}\left\{d_{i}\left(n, c_{i}\right)=i\right\}
$$

Величина $\Gamma_{i}\left(c_{i}\right)$ корректно определена для любого «истинного» значения параметра $\theta_{i}$ п.р. и может интерпретироваться как максимальная вероятность ложного решения при наименее благоприятной альтернативе.

Мы назовем односторонний тест $d_{i}\left(n, c_{i}\right)$ невырожденныл, если $0<\Gamma_{i}\left(c_{i}\right)<1$ для всех $i \in I$.

Для любого $\theta_{i} \in \Theta_{i}$ определим положительное целое число $N_{i}\left(c_{i}\right)$ следуюшим образом:

$$
N_{i}\left(c_{i}\right)=N\left(\Gamma_{i}\left(c_{i}\right)\right)=\min \left\{n: \sum_{k=n}^{\infty} \mathbf{P}_{u_{i}(\cdot)}\left\{\nu_{i}\left(c_{i}\right)=k\right\} \leqslant \Gamma_{i}\left(c_{i}\right)\right\} .
$$

Введем условие

$$
\limsup _{c_{i} \rightarrow \infty} \frac{\ln N_{i}\left(c_{i}\right)}{\left|\ln \Gamma_{i}\left(c_{i}\right)\right|}=0 .
$$

Покажем, что условие (А) выполняется для широкого класса м.о. Рассмотрим последовательность независимых и одинаково распределенных случайных величин $\xi_{i}$ таких, что $\mathbf{E} \xi_{i}=0$ и $\mathbf{E} \exp \left(t \xi_{i}\right)<\infty$ для $|t|<H$. Положим $\eta_{i}=\xi_{i}+a, a<0, \mathbf{E} \xi_{i}^{2}=\sigma^{2}$. 
Для некоторого положительного $c$ рассмотрим решающую функцию $d(n) \stackrel{\text { def }}{=} \mathbb{I}\left(\sum_{k=1}^{n} \eta_{k}>c\right)$ и соответствующий м.о.

$$
\nu \stackrel{\text { def }}{=} \min \left\{n: \sum_{k=1}^{n} \eta_{k}>c\right\} \text {. }
$$

Используя экспоненциальные оценки для сумм независимых случайных величин $([33$, с. 81$])$, получаем

$$
\begin{aligned}
& \mathbf{P}\{\nu=n\} \leqslant \mathbf{P}\{d(n)=1\}=\mathbf{P}\left\{\sum_{k=1}^{n} \eta_{k}>c\right\}=\mathbf{P}\left\{\sum_{k=1}^{n} \xi_{k}>c+n|a|\right\} \\
& \leqslant \begin{cases}\exp \left(-\frac{(c+n|a|)^{2}}{2 n \sigma^{2}}\right), & c+n|a|<H n \sigma^{2}, \\
\exp \left(-\frac{H(c+n|a|)}{2}\right), & c+n|a|>H n \sigma^{2},\end{cases} \\
& \leqslant \exp (-\lambda n),
\end{aligned}
$$

где $\lambda=\min \left(H|a| / 2, a^{2} /\left(2 \sigma^{2}\right)\right)$.

Положим

$$
N(\Gamma) \stackrel{\text { def }}{=} \min \left\{n: \sum_{k=n}^{\infty} \mathbf{P}\{\nu=k\}=\Gamma\right\} .
$$

Тогда из (3.2) следует, что для достаточно малых $Г$ справедливо неравенство $N(\Gamma) \leqslant|\ln \Gamma| / \lambda$. Поэтому

$$
\limsup _{\Gamma \rightarrow 0} \frac{\ln N(\Gamma)}{|\ln \Gamma|}=0
$$

т.е. условие $(\mathrm{A})$ выполнено. В частности, если $\mathbf{P}=\mathbf{P}_{0}, f_{i}(\cdot), i=0,1$, п.р. наблюдений, соответствующих мере $\mathbf{P}_{i}$, и $\eta_{i}=\ln \left(f_{1}\left(x_{i}\right) / f_{0}\left(x_{i}\right)\right)$, мы получаем, что условие (А) выполняется для теста ПТОП.

\section{Основные неравенства.}

Теорема 1. Для любого $\nu_{i}\left(c_{i}\right) \in \mathscr{M}, i \in I$, соответствующего одностороннему невырожденному решающему правилу $d_{i}\left(\cdot, c_{i}\right)$, и любой mочкu $\theta_{i} \in \Theta_{i}$

$$
\mathscr{K}_{i}\left(\nu_{i}\left(c_{i}\right), \theta_{i}\right) \stackrel{\text { def }}{=} \frac{\mathbf{E}_{\theta_{i}} \nu_{i}\left(c_{i}\right)}{\left|\ln \Gamma_{i}\left(c_{i}\right)\right|} \geqslant \mathbf{I}_{i}^{-1}\left(\theta_{i}\right)\left(1-\frac{\ln N_{i}\left(c_{i}\right)}{\left|\ln \Gamma_{i}\left(c_{i}\right)\right|}\right) .
$$

Если $\Gamma_{i}\left(c_{i}\right) \rightarrow 0$, то величину $\mathscr{K}_{i}\left(\nu_{i}\left(c_{i}\right), \theta_{i}\right)$ можно интерпретировать (см. введение) как отношение среднего времени до верного решения при распределении $\mathbf{P}_{\theta_{i}}$ из семейства $\mathscr{P}_{i}$ к логарифму наименьшего среднего времени до ложного решения для значения параметра из множества $\mathscr{U}_{i}$, соответствующего наименее благоприятной для $\theta_{i} \in \Theta_{i}$ альтернативе.

Следовательно, $\mathscr{K}_{i}\left(\nu_{i}\left(c_{i}\right), \theta_{i}\right)$ можно интерпретировать как критерии качества для односторонних последовательных тестов. На наш 
взгляд, эти критерии в содержательном смысле же хуже, чем обычно применяемые критерии в задачах последовательной проверки гипотез, и, как было продемонстрировано во введении, дают возможность также находить оптимальные методы и в стандартных постановках.

Из теоремы 1 следует, что для любого м.о. $\nu_{i}\left(c_{i}\right) \in \mathscr{M}_{i}, i \in I$, и любой (выше определенной) пары $\left(\theta_{i}, u_{i}(\cdot)\right) \in \Theta_{i} \times \mathscr{U}_{i}$ существование пределов $\lim _{c_{i} \rightarrow \infty} \mathscr{K}_{i}\left(\nu_{i}\left(c_{i}\right), \theta_{i}\right)$ (при $\lim \sup _{c_{i} \rightarrow \infty} \Gamma_{i}\left(c_{i}\right)=0$ и выполнении условия (А)) влечет следующие неравенства:

$$
\lim _{c_{i} \rightarrow \infty} \mathscr{K}_{i}\left(\nu_{i}\left(c_{i}\right), \theta_{i}\right) \geqslant \mathbf{I}_{i}^{-1}\left(\theta_{i}\right), \quad i \in I .
$$

Как было показано выше, для широкого класса тестов $N_{i}\left(c_{i}\right) \sim\left|\ln \Gamma_{i}\left(c_{i}\right)\right|$ при $\Gamma_{i}\left(c_{i}\right) \rightarrow 0$.

Неравенства (3.4) указывают априорные нижние границы для введенных критериев. Пределы (при $\lim \sup _{c_{i} \rightarrow \infty} \Gamma_{i}\left(c_{i}\right)=0$ ) этих нижних границ не зависят от конкретного решающего правила, а зависят лишь от пары $\left(\theta_{i}, u_{i}(\cdot)\right)$, соответствующей «истинной» параметрической точке и ее наименее благоприятной альтернативе.

Таким образом, для новых критериев имеет место ситуация, вполне аналогичная ситуации с классическим неравенством Рао-Крамера в теории оценки параметров: мы можем формулировать задачу достижимости априорной нижней границы и поиска методов, для которых эта граница достигается.

Односторонние тесты $\nu_{i}\left(c_{i}\right)$, для которых в (3.4) достигается знак равенства, мы называем адаптивнылми асимптотически оптимальньми. Термин «адаптивный» в данном контексте означает, что асимптотическая оптимальность теста не зависит от истинной (неизвестной статистику) п.р. наблюдений из семейства $\mathscr{P}_{i}$.

Асимптотически оптимальные односторонние тесты. Пусть $a>0$ - произвольное число. Зафиксируем некоторый индекс $i \in I$ и обозначим $\Theta(a) \subset \Theta, \Theta(a)=\left(\Theta_{i}(a) \times \mathscr{U}_{i}(a)\right)$, конечную $1 / a$-сеть в $\Theta$, $\# \Theta(a)=R(a)$.

Для проверки гипотезы $\mathbf{H}_{i}$ введем м.о.

$$
T_{i}\left(c_{i}, a\right) \stackrel{\text { def }}{=} \inf \left\{n: \mathscr{L}_{i}(n, a) \stackrel{\text { def }}{=} \min _{u \in \mathscr{\mathscr { U }}_{i}(a)} \max _{\theta_{i} \in \Theta_{i}(a)} \sum_{k=1}^{n} \ln \frac{f\left(\theta_{i}, x_{k}\right)}{f\left(u, x_{k}\right)}>c_{i}\right\}
$$

и соответствующее решающее правило

$$
\tilde{d}_{i}\left(n, c_{i}, a\right) \stackrel{\text { def }}{=}\left\{\begin{array}{ccc}
i, & \text { если } & \mathscr{L}_{i}(n, a)>c_{i}, \\
-1, & \text { если } & \mathscr{L}_{i}(n, a) \leqslant c_{i} .
\end{array}\right.
$$

Заметим, что в (3.5) можно поменять местами операции max и $\min$. Положим

$$
\Gamma_{i}^{a}\left(c_{i}\right) \stackrel{\text { def }}{=} \sup _{n} \mathbf{P}_{u_{i}(\cdot)}\left\{\widetilde{d}_{i}\left(n, c_{i}, a\right)=i\right\}
$$


(напомним, что $u_{i}\left(\theta_{i}\right)=u_{i}(\cdot)$ есть наименее благоприятная точка, соответствующая точке $\left.\theta_{i}\right)$.

Теорема 2. Пусть $\theta_{i}-$ произвольная точка в $\Theta_{i}$. Тогда для любого $\varepsilon>0$ существуют такие $a(\varepsilon), \rho(\varepsilon)(a(\varepsilon) \uparrow \infty, \rho(\varepsilon) \downarrow 0$ при $\varepsilon \rightarrow 0)$, ито односторонний тест $\widetilde{d}_{i}\left(n, c_{i}, a(\varepsilon)\right) \stackrel{\text { def }}{=} \widetilde{d}_{i}\left(n, c_{i}, \varepsilon\right)$, соответствуюиий моменту остановки $T_{i}\left(c_{i}, a(\varepsilon)\right) \stackrel{\text { def }}{=} T_{i}\left(c_{i}, \varepsilon\right)$, и вероятность ошибки $\Gamma_{i}^{a(\varepsilon)}\left(c_{i}\right) \stackrel{\text { def }}{=} \Gamma_{i}^{\varepsilon}\left(c_{i}\right)$ удовлетворяют следуюшим соотношениям:

$$
\begin{gathered}
\mathbf{I}_{i}^{-1}\left(\theta_{i}\right)+\rho(\varepsilon) \geqslant \lim _{c_{i} \rightarrow \infty} \mathscr{K}_{i}\left(T_{i}\left(c_{i}, \varepsilon\right), \theta_{i}\right) \geqslant \mathbf{I}_{i}^{-1}\left(\theta_{i}\right) ; \\
\liminf _{c_{i} \rightarrow \infty} \frac{\left|\ln \Gamma_{i}^{\varepsilon}\left(c_{i}\right)\right|}{c_{i}} \geqslant 1-\varepsilon .
\end{gathered}
$$

Таким образом, односторонние тесты, соответствующие м.о.

$$
T_{i}\left(c_{i}\right) \stackrel{\text { def }}{=} \inf \left\{n: \min _{u \in \mathscr{U}_{i}} \max _{\theta_{i} \in \Theta_{i}} \sum_{k=1}^{n} \ln \frac{f\left(\theta_{i}, x_{k}\right)}{f\left(u, x_{k}\right)}>c_{i}\right\},
$$

являются адаптивными асимптотически оптимальными и вероятности ложных решений для этих тестов стремятся к нулю с экспоненциальной скоростью при стремлении к бесконечности «большого параметра».

4. Многосторонние тесты: основные результаты. Пусть $c=\left(c_{0}, c_{1}, \ldots, c_{(k-1)}\right)-$ векторный «большой» параметр. Последовательная процедура проверки $k$ гипотез состоит в выборе в момент $n$ из $k+1$ альтернативы: а) прекратить наблюдения и принять гипотезу $\left.\mathbf{H}_{i}, i \in I, \mathrm{~b}\right)$ продолжить наблюдения. Таким образом, решаюшее правило $d_{c}(n)$, зависящее от параметра $c$, имеет вид:

$$
d_{c}(n) \stackrel{\text { def }}{=} \begin{cases}i, i=0,1, \ldots, k-1 & \text { (остановиться и принять } \mathbf{H}_{i} \\ -1 & \text { на шаге } n), \\ & \text { (продолжить наблюдения) } .\end{cases}
$$

Пусть $\theta_{i} \in \Theta_{i}, i \in I,-$ произвольная точка. Отправляясь от $\theta_{i}$, определим точку $u_{i}\left(\theta_{i}\right) \stackrel{\text { def }}{=} u_{i}(\cdot) \in \mathscr{U}_{i} \stackrel{\text { def }}{=} \Theta \backslash \Theta_{i}$ аналогично (3.1). Таким образом, если $\mathbf{P}_{\theta_{i}}$ - главная гипотеза, то ее наименее благоприятная альтернатива в множестве $\mathscr{U}_{i}-$ это гипотеза $\mathbf{P}_{u_{i}(\cdot)}$.

Для любой $\mathbf{H}_{i}, i \in I$, можно определить вероятности ложных решений аналогично односторонним тестам:

$$
\begin{array}{cc}
\alpha_{i j}(c) \stackrel{\text { def }}{=} \sup _{n} \mathbf{P}_{\theta_{i}}\left\{d_{c}(n)=j, j \neq i\right\}, & \beta_{i j}(c) \stackrel{\text { def }}{=} \inf _{n} \mathbf{P}_{\theta_{i}}\left\{d_{c}(n)=j, j \neq i\right\}, \\
\gamma_{i}(c) \stackrel{\text { def }}{=} \sup _{n} \mathbf{P}_{u_{i}(\cdot)}\left\{d_{c}(n)=i\right\}, & \rho_{i}(c) \stackrel{\text { def }}{=} \inf _{n} \mathbf{P}_{u_{i}(\cdot)}\left\{d_{c}(n)=i\right\} .
\end{array}
$$

Мы будем говорить, что тест $d_{c}$ невырожден, если

$$
1>\sum_{j, j \neq i} \alpha_{i j}(c) \geqslant \sum_{j, j \neq i} \beta_{i j}(c)>0, \quad 1>\max _{i} \gamma_{i}(c) \geqslant \min _{i} \gamma_{i}(c)>0 .
$$


Пусть

$$
\tau_{c} \stackrel{\text { def }}{=} \inf \left\{n: d_{c}(n) \neq-1\right\}
$$

- м.о., соответствующий последовательному тесту. Для любого набора $\theta=\left(\theta_{0}, \theta_{1}, \ldots, \theta_{k-1}\right) \in \Theta$ рассмотрим векторный критерий $\mathscr{K}\left(\tau_{c}, \theta\right)=$ $\left\{\mathscr{K}_{i}\left(\tau_{c}, \theta_{i}\right)\right\}_{i \in I}$, где $\mathscr{K}_{i}\left(\tau_{c}, \theta_{i}\right) \stackrel{\text { def }}{=}\left|\ln \gamma_{i}(c)\right|^{-1} \mathbf{E}_{\theta_{i}} \tau_{c}$. Интерпретация величин $\mathscr{K}_{i}\left(\tau_{c}, \theta_{i}\right), i \in I$, точно такая же, как и выше.

На наш взгляд, такой критерий не хуже в содержательном смысле, чем обычно используемые для последовательной проверки гипотез (по тем же соображениям, что и для односторонних тестов).

Для любого $\theta$ определим набор положительных цельх чисел $\left\{M_{i}(c)\right\}$, $i \in I$, следующим образом:

$$
M_{i}(c)=M_{i}\left(\gamma_{i}(c)\right) \stackrel{\text { def }}{=} \min \left\{n: \sum_{k=n}^{\infty} \mathbf{P}_{u_{i}(\cdot)}\left\{\tau_{c}=k\right\} \leqslant \gamma_{i}(c)\right\} .
$$

Введем в рассмотрение следующее условие:

$$
\limsup _{c \rightarrow \infty} \frac{\ln M_{i}(c)}{\left|\ln \gamma_{i}(c)\right|}=0 .
$$

Так же, как и выше, можно показать, что условие (В) выполнено для широкого класса м.о. и, в частности, для двустороннего ПТОП.

Мы рассматриваем м.о. (и соответствуюшие тесты), которые принадлежат классу

$$
\mathscr{N} \stackrel{\text { def }}{=}\left\{\tau_{c}: \text { для любого конечного } c \sup _{\theta_{i} \in \Theta_{i}} \mathbf{E}_{\theta_{i}} \tau_{c}<\infty \forall i \in I\right\} .
$$

Теорема 3. Для любого момента остановки $\tau_{c} \in \mathscr{N}$, соответствующего невырожденному тесту, и любой точки $\theta_{i} \in \Theta_{i}, i \in I$, выполнено неравенство

$$
\begin{aligned}
\mathscr{K}_{i}\left(\tau_{c}, \theta_{i}\right) \geqslant \mathbf{I}_{i}^{-1}\left(\theta_{i}\right)\{ & 1-a_{i}(c)-\left(1-a_{i}(c)+b_{i}(c)\right) \frac{\ln M_{i}(c)}{\left|\ln \gamma_{i}(c)\right|} \\
& +\left(1-a_{i}(c)\right) \frac{\ln \left(1-a_{i}(c)\right)}{\left|\ln \gamma_{i}(c)\right|} \\
& \left.+\frac{b_{i}(c)}{\left|\ln \gamma_{i}(c)\right|} \ln \frac{b_{i}(c)}{1-\rho_{i}(c)}\right\},
\end{aligned}
$$

где $a_{i}(c)=\sum_{j, j \neq i} \alpha_{i j}(c), b_{i}(c)=\sum_{j, j \neq i} \beta_{i j}(c)$.

Из теоремы 3 следует, что для любых точек $\theta_{i} \in \Theta_{i}$ и невырожденных многосторонних тестов существование пределов $\lim _{c \rightarrow \infty} \mathscr{K}_{i}\left(\tau_{c}, \theta_{i}\right)$ и выполнение условия (В) влечет (в предположении, что вероятности ложных решений стремятся к нулю) неравенства

$$
\lim _{c \rightarrow \infty} \mathscr{K}_{i}\left(\tau_{c}, \theta_{i}\right) \geqslant \mathbf{I}_{i}^{-1}\left(\theta_{i}\right), \quad i \in I .
$$


Многосторонние тесты $\tau_{c}$, для которых во всех неравенствах (4.3) достигается равенство (т.е. векторный критерий $\left\{\mathscr{K}_{i}\left(\tau_{c}, \theta_{i}\right)\right\}_{i \in I}$ достигает своей априорной нижней границы для любого набора точек $\left.\left(\theta_{0}, \theta_{1}, \ldots, \theta_{k-1}\right)\right)$, мы называем адаптивными асимптотически оптимальньми. Термин «адаптивный» в этом контексте означает (как и ранее), что свойство асимптотической оптимальности теста не зависит от истинной п.р. наблюдений из семейств $\mathscr{P}_{\theta_{i}}$.

Асимптотически оптимальные многосторонние тесты. Определим м.о.

$$
T(c, a) \stackrel{\text { def }}{=} T_{0}\left(c_{0}, a\right) \wedge T_{1}\left(c_{1}, a\right) \wedge T_{2}\left(c_{2}, a\right) \wedge \cdots \wedge T_{k-1}\left(c_{k-1}, a\right),
$$

где $T_{i}\left(c_{i}, a\right)$ указаны в $(3.5)$.

Пусть $I(T(c, a)) \stackrel{\text { def }}{=}\left\{i \in I: T_{i}\left(c_{i}, a\right)=T(c, a)\right\}$.

Соответствующее решающее правило может быть записано в виде

$$
d_{c}^{*}(n, a)= \begin{cases}i, & \text { если } n=T(c, a)=T_{i}\left(c_{i}, a\right)<T_{j}\left(c_{j}, a\right), \\ & j \neq i, i, j \in I, \\ \text { любое } j, & \text { если } n=T(c, a), \# I(T(c, a))>1, \\ & j \in I(T(c, a)), \\ -1, & \text { если } T(c, a)>n .\end{cases}
$$

Определим также вероятности ложных решений для правила $d_{c}^{*}(n, a)$ :

$$
\begin{aligned}
\alpha_{i j}^{*}(c, a) & \stackrel{\text { def }}{=} \sup _{n} \mathbf{P}_{\theta_{i}}\left\{d_{c}^{*}(n, a)=j, j \neq i\right\}, \\
\beta_{i j}^{*}(c, a) & \stackrel{\text { def }}{=} \inf _{n} \mathbf{P}_{\theta_{i}}\left\{d_{c}^{*}(n, a)=j, j \neq i\right\}, \\
\gamma_{i}^{*}(c, a) & \stackrel{\text { def }}{=} \sup _{n} \mathbf{P}_{u_{i}(\cdot)}\left\{d_{c}^{*}(n, a)=i\right\}, \\
\rho_{i}^{*}(c, a) & \stackrel{\text { def }}{=} \inf _{n} \mathbf{P}_{u_{i}(\cdot)}\left\{d_{c}^{*}(n, a)=i\right\} .
\end{aligned}
$$

Теорема 4. Пусть $\theta_{i}-$ произвольная тоика из $\Theta_{i}$. Тогда для любого $\varepsilon>0$ существуют $a(\varepsilon), r(\varepsilon)(a(\varepsilon) \uparrow \infty, r(\varepsilon) \downarrow 0$ при $\varepsilon \rightarrow 0)$ такие, что многосторонний тест $d_{c}^{*}(n, a(\varepsilon)) \stackrel{\text { def }}{=} d_{c}^{*}(n, \varepsilon)$, порожденный моментом остановки $T(c, a(\varepsilon)) \stackrel{\text { def }}{=} T(c, \varepsilon)$, и вероятности ложных решений $\alpha_{i j}^{*}(c, a(\varepsilon)) \stackrel{\text { def }}{=} \alpha_{i j}^{*}(c, \varepsilon), \gamma_{i}^{*}(c, a(\varepsilon)) \stackrel{\text { def }}{=} \gamma_{i}^{*}(c, \varepsilon)$ удовлетворяют соотношениям

$$
\mathbf{I}_{i}^{-1}\left(\theta_{i}\right)+r(\varepsilon) \geqslant \lim _{c \rightarrow \infty} \mathscr{K}_{i}\left(T(c, \varepsilon), \theta_{i}\right) \geqslant \mathbf{I}_{i}^{-1}\left(\theta_{i}\right)
$$

$u$

$$
\begin{gathered}
\liminf _{c_{j} \rightarrow \infty} \frac{\left|\ln \alpha_{i j}^{*}\left(c_{j}, \varepsilon\right)\right|}{c_{j}} \geqslant 1-\varepsilon, \quad 0 \leqslant i \neq j \leqslant k-1, \\
\liminf _{c_{i} \rightarrow \infty} \frac{\left|\ln \gamma_{i}^{*}\left(c_{i}, \varepsilon\right)\right|}{c_{i}} \geqslant 1-\varepsilon .
\end{gathered}
$$


Таким образом, многосторонний тест, порожденный м.о.

$$
T(c) \stackrel{\text { def }}{=} T_{0}\left(c_{0}\right) \wedge T_{1}\left(c_{1}\right) \wedge T_{2}\left(c_{2}\right) \wedge \cdots \wedge T_{k-1}\left(c_{k-1}\right),
$$

является адаптивньм асимптотически оптимальным и вероятности ложных решений этого теста стремятся к нулю экспоненциально при стремлении «большого параметра» $c$ к бесконечности.

\section{СПИСОК ЛИТЕРАТУРЫ}

1. Wald A. Sequential tests of statistical hypotheses. - Ann. Math. Statist., 1945, v. 16, p. $117-186$.

2. ШІиряев A.Н. Статистический последовательный анализ. М.: Наука, 1976, 272 с.

3. Wald A., Wolfowitz J. Optimum character of the sequential probability ratio test. Ann. Math. Statist., 1948, v. 19, p. 326-339.

4. Kiefer J., Weiss L. Some properties of generalized sequential probability ratio tests. Ann. Math. Statist., 1957, v. 28, p. 57-74.

5. Lai T.L. Optimal stopping and sequential tests which minimize the maximum expected sample size. - Ann. Statist., 1973, v. 1, p. 659-673.

6. Lai T. L. Nearly optimal sequential tests of composite hypotheses. - Ann. Statist., 1988 , v. 16 , № 2 , p. 856-886.

7. Драгалин В. П., Новиков А. А. Асимптотическое решение задачи Кифера-Вейса для процессов с независимыми приращениями. - Теория вероятн. и ее примен., 1987 , т. 32 , в. 4 , с. $679-690$.

8. Hoeffding $W$. Lower bounds for the expected sample size and the average risk of a sequential procedure. - Ann. Math. Statist., 1960, v. 31, p. 352-368.

9. Lorden G. 2-SPRT's and the modified Kiefer-Weiss problem of minimizing an expected sample size. - Ann. Statist., 1976, v. 4, № 2, p. 281-291.

10. Bartlett M. S. The large-sample theory of sequential tests. - Proc. Cambridge Philos. Soc., 1946, v. 42 , p. $239-244$.

11. Cox D. R. Large sample sequential tests for composite hypotheses. - Sankhyā, 1963, v. 25 , p. $5-12$.

12. Joanes D.N. Sequential tests of composite hypotheses. - Biometrika, 1972, v. 59, p. $633-637$.

13. Gombay E. Sequential testing of composite hypotheses. - Limit Theorems in Probability and Statistics (1999, Balatonlelle), v. II. Budapest: János Bolyai Math. Soc., 2002, p. 107-125.

14. Cox C. P., Roseberry T.D. A large sample sequential test, using concomitant information, for discrimination between two composite hypotheses. - J. Amer. Statist. Assoc., 1966, v. 61, p. 357-367.

15. Айвазян C.A. Сравнение оптимальных свойств критериев Неймана-Пирсона и Вальда. - Теория вероятн. и ее примен., 1959, т. 4, в. 1, с. 86-93.

16. Dragalin V.P., Novikov A. A. Adaptive sequential tests for composite hypotheses. Обозрение прикл. промышл. матем., 1999, т. 6, в. 2, с. 387-398.

17. Lorden $G$. Nearly-optimal sequential tests for finitely many parameter values. - Ann. Statist., 1977, v. 5, № 1, p. 1-21.

18. Павлов И.В. Последовательная процедура различения многих сложных гипотез. - Теория вероятн. и ее примен., 1987 , т. 32 , в. 1, с. 149-153.

19. Dragalin V.P., Tartakovsky A.G., Veeravalli V.V. Multihypothesis sequential probability ratio tests. I. Asymptotic optimality. - IEEE Trans. Inform. Theory, 1999, v. 45 , p. $2448-2461$.

20. Dragalin V. P., Tartakovsky A. G., Veeravalli V.V. Multihypothesis sequential probability ratio tests. II. Accurate asymptotic expansions for the expected sample size. IEEE Trans. Inform. Theory, 2000, v. 46, № 4, p. 1366-1383. 
21. Фишман М.М. Асимптотически оптимальные многоальтернативные последовательные процедуры для различения процессов, минимизирующие среднюю длительность наблюдений. - Радиотехника и электроника, 1984, т. 29, № 8, с. 15161524.

22. Голубев Г.К., Хасьминский Р. З. О последовательном различении нескольких сигналов в гауссовском белом шуме. - Теория вероятн. и ее примен., 1983, т. 28, B. 3 , c. $544-554$.

23. Baum C. W., Veeravalli $V$. V. A sequential procedure for multihypothesis testing. IEEE Trans. Inform. Theory, 1994, v. 40, №6, p. 1994-2007.

24. Armitage $P$. Sequential analysis with more than two alternative hypotheses, and its relation to discriminant function analysis. - J. Roy. Statist. Soc. Ser. B, 1950, v. 12, p. 137-144.

25. Tartakovsky A. G. Asymptotic optimality of certain multihypothesis sequential tests: non-i.i.d. case. - Stat: Inference Stoch. Process., 1998, v. 1, № 3, p. 265-295.

26. Tartakovsky A. G., Li X. R., Yaralov G. Sequential detection of targets in multichannel systems. - IEEE Trans. Inform. Theory, 2003, v. 49, № 2, p. 425-445.

27. Lai T. L. Sequential multiple hypothesis testing and efficient fault detection-isolation in stochastic systems. - IEEE Trans. Inform. Theory, 2000, v. 46, № 2, p. 595-608.

28. Simons $G$. Lower bounds for average sample number of sequential multihypothesis tests. - Ann. Math. Statist., 1967, v. 38, p. 1343-1364.

29. Бродский Б. Е., Дарховский Б. С. Сравнительный анализ некоторых непараметрических методов скорейшего обнаружения момента «разладки» случайной последовательности. - Теория вероятн. и ее примен., 1990, т. 35, в. 4, с. 655-668.

30. Brodsky B. E., Darkhovsky B.S. Non-parametric Statistical Diagnosis: Problems and Methods. Dordrecht: Kluwer, 2000, 452 p.

31. Дарховский Б. С., Бродский Б. Е. Непараметрический метод скорейшего обнаружения изменения среднего случайной последовательности. - Теория вероятн. и ее примен., 1987, т. 32, в. 4, с. 703-711.

32. Бродский Б. Е., Дарховский Б. С. Асимптотически оптимальные методы последовательной проверки сложных гипотез. - Докл. РАН, 2006, т. 408, № 1, с. 11-15

33. Петров В. В. Предельные теоремы для сумм независимых случайных величин. M.: Наука, 1987,317 c.

34. Woodroofe M. Nonlinear Renewal Theory in Sequential Analysis. Philadelphia: SIAM, $1982,119 \mathrm{p}$. 